\title{
SIMPLE MODELS FOR EXPLOITATIVE AND INTERFERENCE COMPETITION
}

\section{A.L. JENSEN}

School of Natural Resources, University of Michigan, Ann Arbor, MI $48109-1115$ (U.S.A.) (Accepted 13 February 1986)

\begin{abstract}
Jensen, A.L., 1987. Simple models for exploitative and interference competition. Ecol. Modelling, 35: 113-121.

Competition is exploitative when species compete for the same limited resource, and interference when species deplete one another's resources by interferences such as aggressive displays or fighting. If pure exploitative competition is defined as an effect on the carrying capacity, and if pure interference competition is defined as an effect on the rate of increase per individual, then the logistic equation can be modified to describe both pure exploitative and pure interference competition. Both models have identical equilibrium properties and very similar trajectories; it would be difficult to distinguish between these two types of competition using only data on abundances. However, for pure interference competition the relation between the rate of change per individual of one species and abundance of the second is linear, whereas for pure exploitative competition the relation between the rate of change per individual of one species and abundance of the second is non-linear; this is an important consideration when exploiting competitive species.
\end{abstract}

\section{INTRODUCTION}

Ecologists have long recognized that there are different forms of competition, and Park (1954) distinguished exploitative competition from interference competition. Exploitative competition is the joint exploitation of a common resource. Population densities are expressible directly as units of the resource, and competitive ability is the rate of resource consumption (Gill, 1974). As an extreme example, one species may feed on a plant during the day, and another species may feed on the same plant during the night (Krebs, 1971). Each species diminishes the resource for the other, but there is no direct contact or conflict. In interference competition individuals deplete one another's resource supply by interferences such as aggressive displays, fighting, and collisions (Schoener, 1976). 
At least two models have been developed to describe exploitative and interference competition (MacArthur, 1972; Schoener, 1976). In both of these models competition was developed in terms of a competitive utilization of an energy or resource supply. The models of MacArthur (1972) and Schoener (1976) offer a wider range of behavior than the Lotka-Volterra model and they may be necessary to describe the behavior of many species, but they are more complex than the Lotka-Volterra model and they are not unique; many different model forms are possible.

A different approach that maintains the simplicity of the Lotka-Volterra equations is to model interference competition with the conventional Lotka-Volterra equations and to model exploitative competition with a similar model in which each species impacts on the carrying capacity of the other species. To indicate the limited scope of the models developed, I will use the terms pure interference and pure exploitative competition. Pure interference competition is competition in which species interact in such a way that individuals of one species are equivalent to a certain number of individuals of another species. Pure exploitative competition is competition in which one species reduces the capacity of the environment to support another species by acting on the carrying capacity of that species. The models are developed for only two species, but they can be extended to any number of species.

PURE INTERFERENCE COMPETITION: THE LOTKA AND VOLTERRA EQUATIONS

The Lotka-Volterra equations are well known and are the type model for competition in most introductory and mathematical ecology textbooks (e.g., Volterra, 1926; Lotka, 1932; Slobodkin, 1961; Pielou, 1969; Vandermeer, 1969, 1981; Goel et al., 1971; MacArthur, 1972; Krebs, 1972; Williamson, 1972; Pianka, 1974; Emlen, 1977; Roughgarden, 1979; Ricklefs, 1979; Freedman, 1980). The equations are:

$$
\begin{aligned}
& \frac{1}{N_{1}} \frac{\mathrm{d} N_{1}}{\mathrm{~d} t}=r_{1}-r_{1} N_{1} / K_{1}-\alpha_{12} r_{1} N_{2} / K_{1} \\
& \frac{1}{N_{2}} \frac{\mathrm{d} N_{2}}{\mathrm{~d} t}=r_{2}-r_{2} N_{2} / K_{2}-\alpha_{21} r_{2} N_{1} / K_{2}
\end{aligned}
$$

where $r_{i}$ is the intrinsic rate of increase of species $i, r_{i} N_{i} / K_{i}$ is the inhibitory effect of species $i$ on species $i$, and $r_{i} \alpha_{i j} N_{j} / K_{i}$ is the inhibitory effect of species $j$ on species $i$. Written in the above form the competition coefficients $\alpha_{12}$ and $\alpha_{21}$ measure interspecific competition relative to intraspecific competition. 


\section{PURE EXPLOITATIVE COMPETITION}

If competition is pure exploitative rather than pure interference, it can be modelled as a decrease in the resources available for growth. It is assumed that growth of each species alone is described by the logistic equation. The environment can support $K_{i}$ individuals of species $i$ without species $j$. In the presence of species $j$, the capacity of the environment to support species $i$ is reduced by the amount $\alpha_{i j} N_{j}$, where $\alpha_{i j}$ is the resource per unit time necessary for each individual of species $j$ relative to the carrying capacity for species $i$. Under these assumptions the equations for pure exploitative competition are:

$$
\begin{aligned}
& \frac{1}{N_{1}} \frac{\mathrm{d} N_{1}}{\mathrm{~d} t}=r_{1}-\frac{r_{1} N_{1}}{K_{1}-\alpha_{12} N_{2}} \\
& \frac{1}{N_{2}} \frac{\mathrm{d} N_{2}}{\mathrm{~d} t}=r_{2}-\frac{r_{2} N_{2}}{K_{2}-\alpha_{21} N_{1}}
\end{aligned}
$$

The equilibrium properties of the pure exploitative model are exactly the same as those of the pure interference model, and at equilibrium, where $\mathrm{d} N_{1} / \mathrm{d} t=\mathrm{d} N_{2} / \mathrm{d} t=0$, the relations between the saturation levels of $N_{1}$ and $N_{2}$ are given by the equations:

$N_{1}=K_{1}-\alpha_{12} N_{2}$

$N_{2}=K_{2}-\alpha_{21} N_{1}$

Depending on the relations among $K_{1}, K_{2}, \alpha_{12}$, and $\alpha_{21}$, these equations indicate that competition will result in one of four different outcomes and these outcomes are discussed in detail for the Lotka-Volterra model by, for example, Roughgarden (1979).

\section{EMPIRICAL COMPARISON OF MODELS}

To compare the two models, they were applied to the classic protozoan study of Gause (1934). The data used were for Paramecium caudatum and $P$. aurelia grown in a buffered medium and "half-hoop" concentration of bacteria. Gause (1934) gives the following parameter estimates for $P$. aurelia (species 1) and $P$. caudatum (species 2): $r_{1}=1.124, K_{1}=105, r_{2}=0.794$, and $K_{2}=64$. The competition coefficients varied during the experiments; Gause (1934) gave the estimate for $P$. caudatum $\left(\alpha_{12}\right)$ as 1.64 after the second day and the estimate for $P$. aurella $\left(\alpha_{21}\right)$ as 0.61 after the fourth day. The trajectories were sensitive to the competition coefficients and to obtain the best fit of the models these parameters were adjusted interactively using a computer to minimize the residual sums of squares (non-linear least 
squares). For both models $\alpha_{12}=1.10$. For the pure interference model $\alpha_{21}=0.75$, and for the pure exploitative model $\alpha_{21}=0.60$.

\section{RESULTS AND DISCUSSION}

The models both fit the data well (Figs. 1 and 2), and indeed there is little difference between the trajectories of the two models. The peak of the $P$. caudatum growth curve is more pronounced for the pure exploitative model than for the pure interference model. In terms of the impact on the abundances of the species, there is little difference between pure interference and pure exploitative competition. This result is of importance for it shows that the nature of competition cannot be determined only with abundance data; identification of the nature of competition requires study of the interactions.

The relations between the rate of change per individual for $P$. aurelia (species 1) and abundance of $P$. caudatum (species 2) for $N_{1}=52.5$ is illustrated in Fig. 3 for both the Lotka-Volterra model (pure interference) and the pure exploitative model. The relation is linear for the Lotka-Volterra model and non-linear for the pure exploitative model. If the population of $P$. caudatum is large, its impact on the rate of increase per individual of $P$. aurelia is much greater with the pure exploitative model than with the pure interference model. However, if the population of $P$. caudatum is small, its impact on $P$. aurelia is slightly less with the pure exploitative model. The

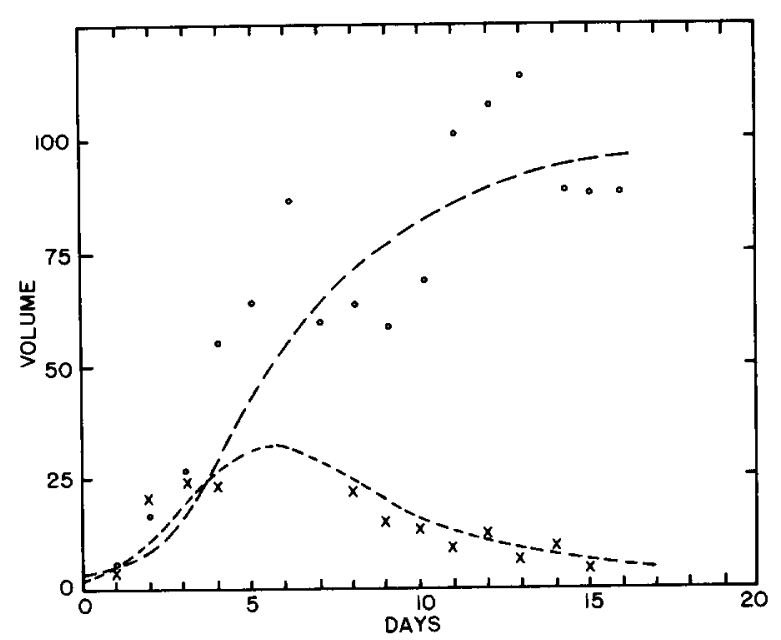

Fig. 1. Observations for growth of Paramecium caudatum (lower curve and x's) and P. aurelia (upper curve and o's) and the fitted Lotka-Volterra (interference) model. 


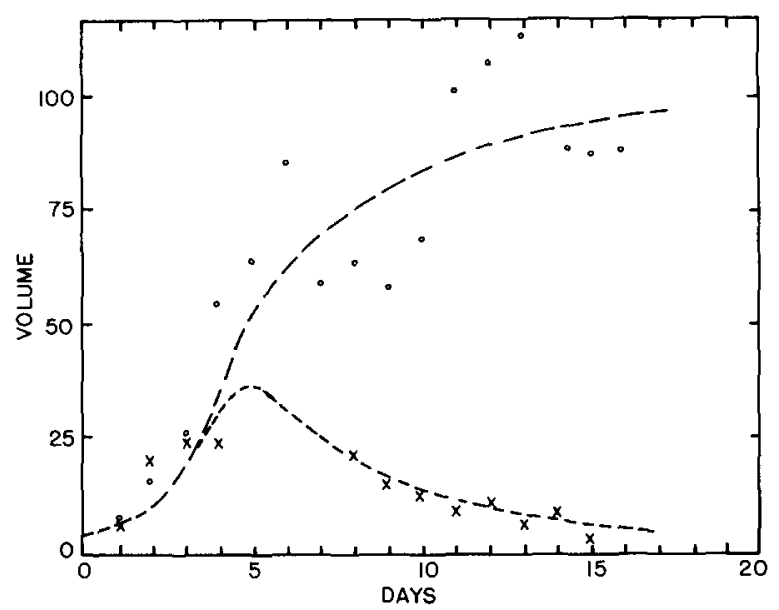

Fig. 2. Observations for growth of Paramecium caudatum (lower curve and x's) and P. aurelia (upper curve and o's) and the fitted exploitative model.

relations between the rate of change per individual for $P$. caudatum (species 2) and abundance of $P$. aurelia (species 1) for $N_{2}=32$ is illustrated in Fig. 4. Large populations of $P$. aurelia cause a much larger decrease in the rate of

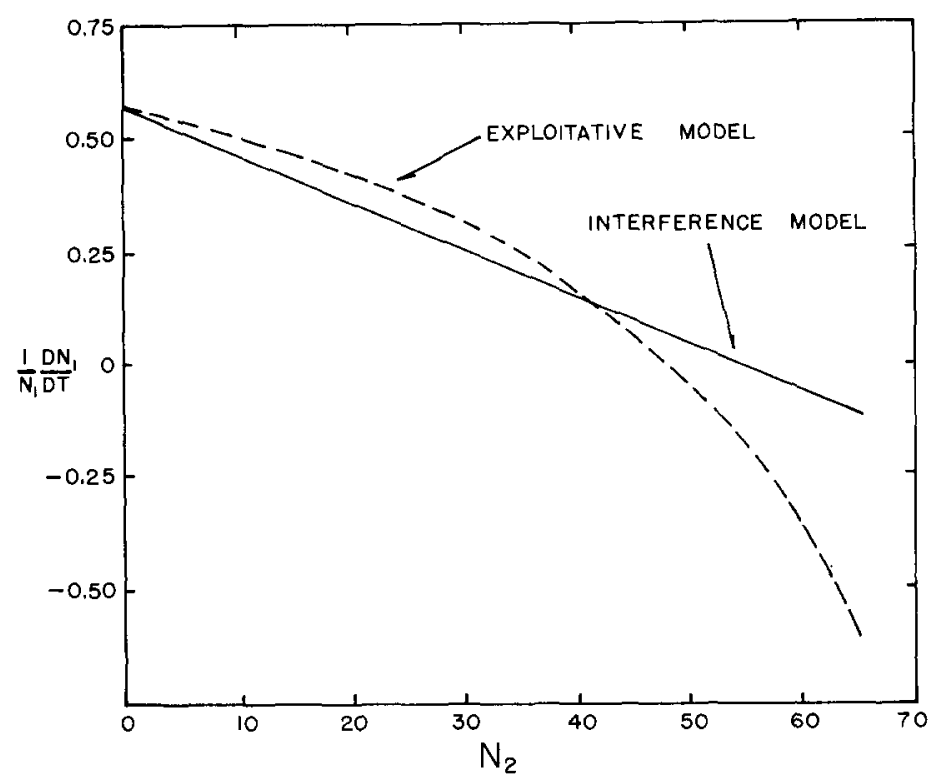

Fig. 3. Relation between rate of change per individual of $P$. aurelia (species 1 ) and the abundance of $P$. caudatum (species 2) for the Lotka-Volterra interference model and for the exploitative model with $N_{1}=52.5$. 


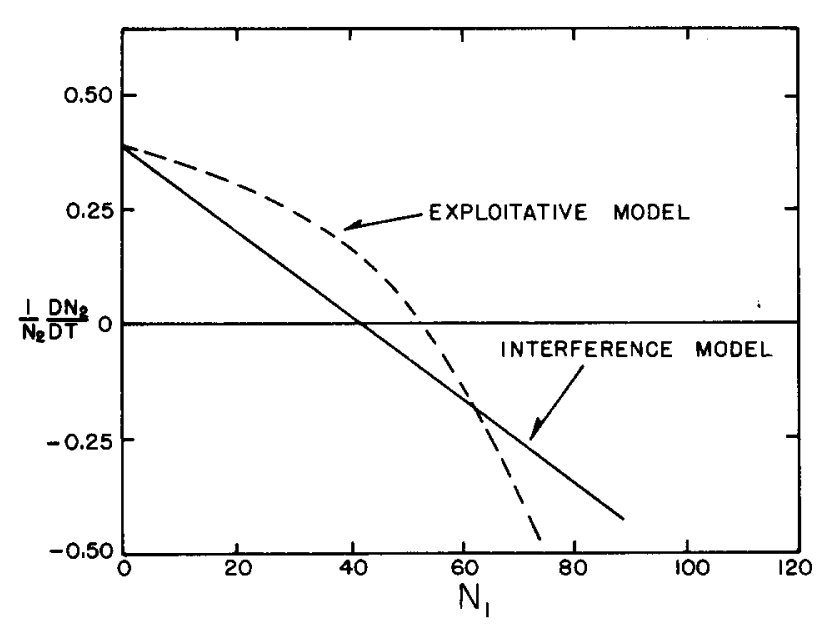

Fig. 4. Relation between rate of change per individual of $P$. caudatum (species 2 ) and the abundance of $P$. aurelia (species 1) for the Lotka-Volterra interference model and for the exploitative model with $N_{2}=32$.

increase per individual of $P$. caudatum with the pure exploitative model than with the pure interference model.

In the pure interference competition model the effects of both species on the rates of increase per individual are additive. For example, the effect of species 1 on its own rate of increase per individual is $r_{1} N_{1} / K_{1}$ and the effect of species 2 on the rate of increase per individual of species 1 is $\alpha_{12} N_{2}$.

In the pure exploitative competition model, the effect of each species on its own rate of growth per individual is additive but the effect of each species on its competitor is not additive. One species is not represented in terms of an equivalent number of the other species. In pure exploitative competition the interaction between species is limited to impacts on the carrying capacities.

An important difference between the two models is that, for the Lotka-Volterra model (pure interference), the rate of change per individual of one species decreases linearly with increase in abundance of the second species and this has been a point of criticism (Roughgarden, 1979). In the pure exploitative model the rate of change per individual of each species is a curvilinear function of abundance of the other species.

The Lotka-Volterra model has been applied to investigate optimal exploitation of competitive species (Larkin, 1963), and this approach assumes interference competition. The effect of one competitive species on the sustainable yield of a second species depends on the form of competition. Models for yield assessment based on the logistic equation assume that 




Fig. 5. Relation between rate of change, which would be proportional to yield in an exploited species, and abundance of species 1 for 4 different abundances of species 2 for Lotka-Volterra model.

populations have a capacity for increase that is a function of population size and that the capacity for increase is a maximum at an intermediate population size (e.g., Jensen, 1976, 1978). The sustainable yield is proportional to the capacity of a population to increase. The competition models fitted to Gause's protozoan data were applied to obtain the relations between the rate of increase for species 1 and abundance of species 1 in the presence of different abundances of species 2 (Figs. 5 and 6). If these were exploited species yield would be proportional to the rate of increase. Interactive competition has a more severe impact than exploitative competition on the capacity of species 1 to support a fishery (Figs. 5 and 6).

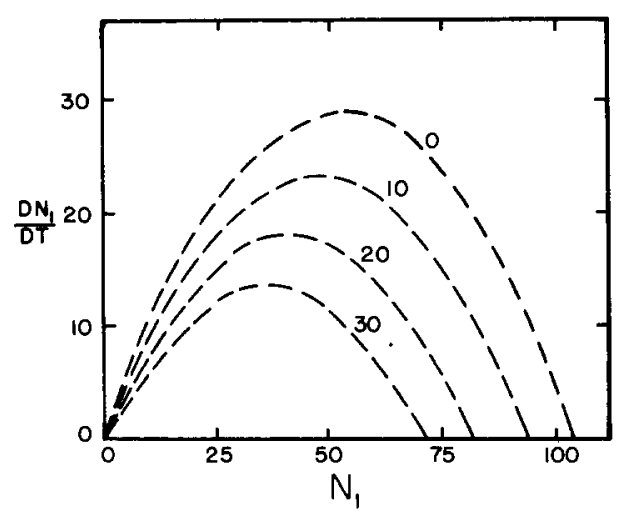

Fig. 6. Relation between rate of change, which would be proportional to yield in an exploited species, and abundance of species 1 for 4 different abundances of species 2 for exploitative model. 


\section{ACKNOWLEDGMENT}

I thank John Vandermeer and Gary Belovsky for reading an earlier draft and providing me with many helpful comments.

\section{REFERENCES}

Ayala, F.J., Gilpin, M.E. and Ehrenfeld, J.G., 1973. Competition between species: theoretical models and experimental tests. Theor. Popul. Biol., 4: 331-356.

Emlen, J.M., 1977. Ecology: An Evolutionary Approach. Addison-Wesley, Reading, MA, 493 pp.

Freedman, H.I., 1980. Deterministic Mathematical Models in Population Ecology. Marcel Dekker, New York, 254 pp.

Gause, G.F., 1934. The Struggle for Existence. Reprinted in 1964 by Hafner, New York, 162 pp.

Gill, D.E., 1974. Intrinsic rate of increase, saturation density, and competitive ability. II. The evolution of competitive ability. Am. Nat., 108: 103-116.

Goel, N.S., Maitra, S.C. and Montroll, E.W., 1971. Nonlinear Models of Interacting Populations. Academic Press, New York, 145 pp.

Jensen, A.L., 1976. Assessment of the United State's lake whitefish (Coregonus clupeaformis) fisheries of Lake Superior, Lake Michigan, and Lake Huron. J. Fish. Res. Board Can., 33: 747-759.

Jensen, A.L., 1978. Assessment of the lake trout fishery in Lake Superior: 1929-1950. Trans. Am. Fish. Soc., 107: 543-549.

Krebs, C.J., 1972. Ecology. Harper \& Row, New York, 694 pp.

Larkin, P.A., 1963. Interspecific competition and exploitation. J. Fish. Res. Board Can., 20: 647-678.

Lotka, A.J., 1932. The growth of mixed populations: two species competing for a common food supply. J. Wash. Acad. Sci., 21: 461-469.

MacArthur, R., 1972. Geographical Ecology: Patterns in the Distribution of Species. Harper \& Row, New York, 269 pp.

Park, T., 1954. Experimental studies of interspecies competition. II. Temperature, humudity, and competition of two species of Tribolium. Physiol. Zool., 27: 177-238.

Pianka, E.R., 1974. Evolutionary Ecology. Harper \& Row, New York, 356 pp.

Pielou, E.C., 1969. An Introduction to Mathematical Ecology. Wiley-Interscience. New York, $286 \mathrm{pp}$.

Pielou, E.C., 1974. Population and Community Ecology. Gordon \& Breach, New York, 424 pp.

Ricklefs, R.E., 1979. Ecology. Chiron Press, New York, 966 pp.

Roughgarden, J., 1979. Theory of Population Genetics and, Evolutionary Ecology: An Introduction. MacMillan, New York, $634 \mathrm{pp}$.

Schoener, T.W., 1976. Alternatives to Lotka-Volterra competition: models of intermediate complexity. Theor. Popul. Biol., 10: 309-333.

Slobodkin, L.B., 1961. Growth and Regulation of Animal Populations. Holt, Rinehart and Winston, New York, $184 \mathrm{pp}$.

Vandermeer, J.H., 1969. The competitive structure of communities: an experimental approach with protozoa. Ecology, 50: 362-371. 
Vandermeer, J.H., 1981. Elementary Mathematical Ecology, John Wiley \& Sons, New York, 294 pp.

Volterra, V., 1926. Fluctuations in the abundance of a species considered mathematically. Nature, 118: 558-560.

Williamson, M., 1972. The Analysis of Biological Populations. Edward Arnold, New York, $180 \mathrm{pp}$. 